\title{
Matrix metalloproteinase 2 and tissue inhibitor of matrix metalloproteinases 2 in the diagnosis of colorectal adenoma and cancer patients
}

\author{
Magdalena Groblewska $^{1}$, Barbara Mroczko ${ }^{1}$, Mariusz Gryko², Bogusław Kędra² \\ and Maciej Szmitkowski ${ }^{1}$
}

${ }^{1}$ Department of Biochemical Diagnostics, ${ }^{2}$ Second Department of General Surgery, Medical University, Białystok, Poland

\begin{abstract}
The aim of the study was to assess the importance of the measurement of matrix metalloproteinase 2 (MMP-2) and tissue inhibitor of matrix metalloproteinases 2 (TIMP-2) in patients with colorectal cancer (CRC) in relation to clinicopathological features of tumor and patients' survival. Additionally, we determined serum MMP-2 and TIMP-2 in colorectal adenoma (CA) patients and healthy controls and compared them with tumor markers, CEA and CA 19-9. The serum levels of MMP-2 and TIMP-2 in 91 CRC patients, 28 CA subjects and 91 healthy controls were determined by ELISA method, but concentrations of CEA and CA 19-9 using MEIA method. Nonparametric statistical analyses were used. Serum levels of MMP-2 and TIMP-2 were significantly lower in CRC patients than in healthy subjects and decreased with tumor stage. Additionally, MMP-2 concentrations were significantly lower in patients with CRC than in CA group. Diagnostic sensitivity of TIMP-2 (59\%) was the highest among biomarkers tested and increased in combined use with CEA (79\%). Moreover, the area under ROC curve (AUC) of TIMP-2 was larger than AUC of MMP-2 in differentiation between CRC and healthy subjects, but lower than AUC of matrix metalloproteinase 2 in differentiation between colorectal cancer and adenoma. Our findings suggest clinical usefulness of TIMP-2 as a biomarker in the diagnosis of CRC, especially in combination with CEA. However, further investigation is necessary.
\end{abstract}

Key words: colorectal cancer, colorectal adenoma, matrix metalloproteinase 2, tissue inhibitor of matrix metalloproteinases 2

\section{Introduction}

The largest risk of colorectal cancer (CRC) development, one of the most frequent malignant tumors in the industrialized world [1], is observed in patients with genetic predispositions or with sporadic adenomatous polyps [2]. The process of colorectal carcinogenesis is complex and long-term and includes several steps of malignant transformation from normal epithelium to cancer cells, which involves numerous genetic changes and results in various phenotypic alterations [3]. The colorectal adenomas are neoplastic tumors with a potential to develop into invasive adenocarcinoma of colon or rectum.

Correspondence: B. Mroczko, Dept. of Biochemical Diagnostics, Medical University, Białystok, Waszyngtona Str. 15a, 15-269 Białystok, Poland; tel.: (+4885) 7468587, fax.: (+4885) 7468585, e-mail:mroczko@umwb.edu.pl
Matrix metalloproteinases (MMPs) are zincdependent endopeptidases which are able to degrading the components of extracellular matrix. Type IV collagen is an important protein of basement membrane. Among all the MMPs, only gelatinases, such as matrix metalloproteinase 2 (MMP-2) and matrix metalloproteinase 9 (MMP-9) participate in degradation of type IV collagen [4-6]. Remodelling of normal and tumoral tissue may be a result of imbalance between MMPs and their natural inhibitors - tissue inhibitors of matrix metalloproteinases (TIMPs). It has been shown that degradation of basement membrane and invasion of epithelium is the first step of tumor development and metastasis [4]. Matrix metalloproteinase 2 (MMP-2) and tissue inhibitor of matrix metalloproteinases 2 (TIMP-2) are involved in tumor invasion and metastasis including breast, gastric, pancreatic and colorectal cancer [7-10].

It was shown that MMP-2 gene expression level was lower in colorectal cancer tissue than in adjacent normal 
mucosa and correlated with the depth of invasion, venous invasion and presence of liver metastasis [11]. Waas et al. showed that plasma MMP-2 levels were lower in CRC metastatic liver disease than in healthy controls [12]. However, Wu et al. revealed that expression of MMP-2 protein significantly increased in CRC tissues but it was not detected in normal colorectal tissues [13].

Li et al. indicated that the balance between MMP2 and TIMP-2 might play a crucial role in the process of colorectal carcinoma invasion and metastasis [14]. They demonstrated that expression of MMP-2 in CRC tissues was significantly higher, but TIMP-2 - significantly lower than in normal tissues. Additionally, they revealed that MMP-2/TIMP-2 ratio was higher in CRC than in normal tissues and gradually decreased with the progression of invasion depth, lymph node metastasis and tumor Duke's stage [14].

It is suggested that MMPs and TIMPs might play a role not only in colorectal tumor invasion and initiation of metastatic cascade, but also in colorectal carcinogenesis from adenomatous polyps. The mRNA and protein expression levels of MMP-2 were investigated in colonic adenoma-carcinoma sequence, ie. normal colonic tissue, colon adenoma, early colon cancer and advanced colon cancer tissues collected from the same subjects [15]. However, little is known about serum levels of MMP-2 and TIMP-2 in colorectal adenoma and cancer patients.

Therefore, the aim of the present study was to investigate the clinical importance of the measurement of serum levels of MMP-2 and TIMP-2 in patients with colorectal adenoma and cancer, especially in the diagnosis of CRC patients as well as in the differentiation between $\mathrm{CA}$ and cancer. We determined the concentrations of MMP-2 and TIMP-2 in the sera of CRC patients in relation to clinico-pathological features of cancer and serum levels of classical tumor markers: carcinoembryonic antigen (CEA) and carbohydrate antigen (CA 19 9). Moreover, we assessed the diagnostic sensitivity and areas under ROC curves (AUC) for all the proteins tested as well as their prognostic significance in CRC patients' survival.

\section{Material and methods}

Patients. The study included a total number of 210 subjects: 91 previously untreated colorectal cancer (CRC) patients (37 women and 54 men, aged 41-84 years) diagnosed and operated on by Second General Surgery Department of the Białystok Medical University Hospital, 28 patients with colorectal adenomas (CA) (10 women and 18 men, aged 32-78 years) and 91 healthy subjects (60 women and 31 men, aged 21-65 years).

The clinical diagnosis of CRC or CA was confirmed by microscopic examination of the material obtained during colonoscopy and/or surgery. Eighty-five CRC patients underwent surgical tumor resection, while six patients had non-resectable tumors. The staging was based on a routine histopathological analysis and clinical assessment, according to Duke's classification. The tumors were classified in accordance with the staging of the 5th International Union Against Cancer [16]. The subjects who had suffered a heart attack or heart failure were not included in the study. Moreover, the patients with extraintestinal tumors and those after preoperative radio-chemotherapy were excluded from the study.

For the analysis, the CRC patients were divided into five groups: 2 cancer patients in stage A, 5 patients in stage B1, 30 patients in stage $\mathrm{B} 2,25$ patients in stage $\mathrm{C} 1,4$ patients in stage $\mathrm{C} 2$, and 25 patients in stage $\mathrm{D}$, and then sub-divided into: four groups depending on the infiltration of the bowel wall (T1, T2, T3, and T4), four groups depending on nodal involvement (N0, N1, N2 and N3), two groups depending on the presence of distant metastases (M0 and M1). The number of patients in the analyzed subgroups is shown in Table 1.

Protein analyses. Blood samples from all the patients were drawn before surgery or polypectomy. None of the CRC patients had received chemo- or radiotherapy before blood sample collection. To standardize clotting conditions, all sera were separated within 1 hour after blood collection and stored at $-80^{\circ} \mathrm{C}$ until assayed.

Serum levels of MMP-2 and TIMP-2 were measured using enzyme-linked immunosorbent assay kits (ELISA) (R\&D Systems, Abingdon, England) according to the manufacturer's instructions. The serum samples were diluted 10 -fold before determination of MMP-2 and 50-fold before measurement of TIMP-2. The intra-assay coefficient of variation (CV\%) of MMP-2 is reported by the manufacturer to be $5.8 \%$ at a mean concentration of $18.9 \mathrm{ng} / \mathrm{mL}, \mathrm{SD}=1.1$ and of TIMP- 2 - to be $4.4 \%$ at a mean concentration of $1.23 \mathrm{ng} / \mathrm{mL}, \mathrm{SD}=0.054$.

Serum concentrations of CEA and CA 19-9 were measured by microparticle enzyme immunoassay kits (MEIA) (Abbott, Chicago, Illinois). The intra-assay CV for CEA is reported by the manufacturer of the assay kits to be $4.9 \%$ at a mean concentration of 2.2 $\mathrm{ng} / \mathrm{mL}, \mathrm{SD}=0.11$ and the intra-assay CV\% for CA $19-9-4.7 \%$ at a mean concentration of $38.2 \mathrm{IU} / \mathrm{mL}, \mathrm{SD}=1.80$.

The cut-off points for serum levels of MMP-2 $(160 \mathrm{ng} / \mathrm{mL})$ and TIMP-2 $(85 \mathrm{ng} / \mathrm{mL})$ were determined using Microsoft Office Excel software. In the ROC report generated by this program, the cut-off values corresponded to the highest accuracy (minimal false-negative and false-positive results). The positive results of MMP-2 and TIMP2 are below cut-off values. The reference cut-off values for tumor markers (the 95th percentile) were established previously in our department by examining blood sera of healthy volunteers [17]. The cut-off points were $4.0 \mathrm{ng} / \mathrm{mL}$ for CEA and $30.0 \mathrm{IU} / \mathrm{mL}$ for CA 19-9.

Ethical issues. The study was approved by the local ethics committee. All the patients gave their informed consent to participate in the study.

Statistical analysis. A preliminary statistical analysis $(\chi 2$ test) revealed that serum levels of MMP-2, TIMP-2, CEA and CA 19-9 did not follow a normal distribution. Therefore, nonparametric statistical analyses were used. The stages A, B1 and B2 were analyzed as one group (stage $\mathrm{A}+\mathrm{B}$ ) whereas stages $\mathrm{C} 1$ and $\mathrm{C} 2$ - as stage $\mathrm{C}$ because of small numbers of patients in the particular subgroups. Similarly, the $\mathrm{T} 1$ and $\mathrm{T} 2$ patients were analyzed as $\mathrm{T} 1+\mathrm{T} 2$ subgroup.

The Mann-Whitney U-test was used to compare the two groups in each category (colon versus rectal cancer; M0 versus M1 group; resectable tumors versus nonresectable; the group of patients who survived versus patients who died of CRC). Differences between more than two groups (e.g. CRC, CA and healthy controls; stage $\mathrm{A}+\mathrm{B}, \mathrm{C}, \mathrm{D}$; or T1+T2, T3, T4; or N0, N1, N2, N3) were compared using ANOVA on ranks (Kruskal-Wallis tests). When significant differences were found, the post hoc Dwass-Steele-CritchlowFligner test was conducted to determine which groups were different. The Spearman rank correlation test was employed for the cor- 


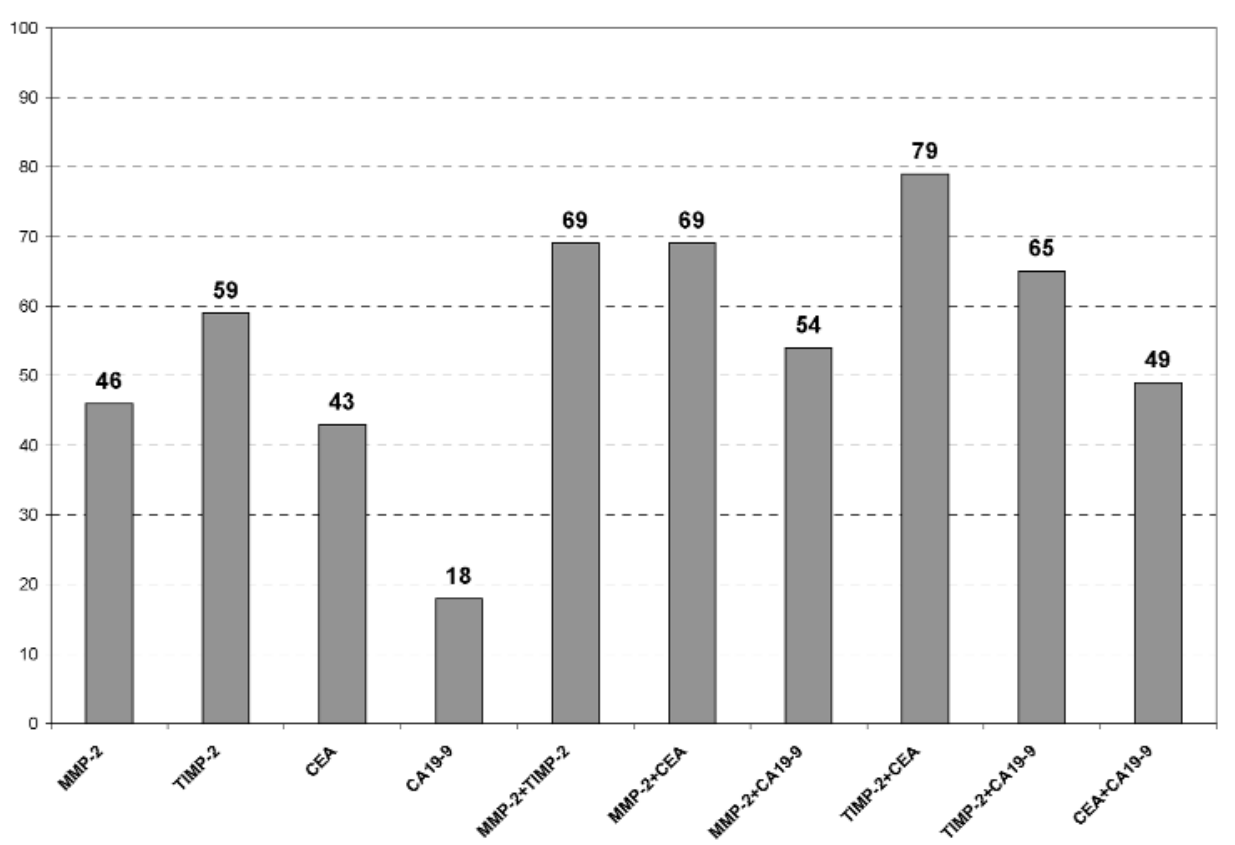

Fig. 1. Percentage of true positive results of MMP-2, TIMP-2 and tumor markers in colorectal cancer patients

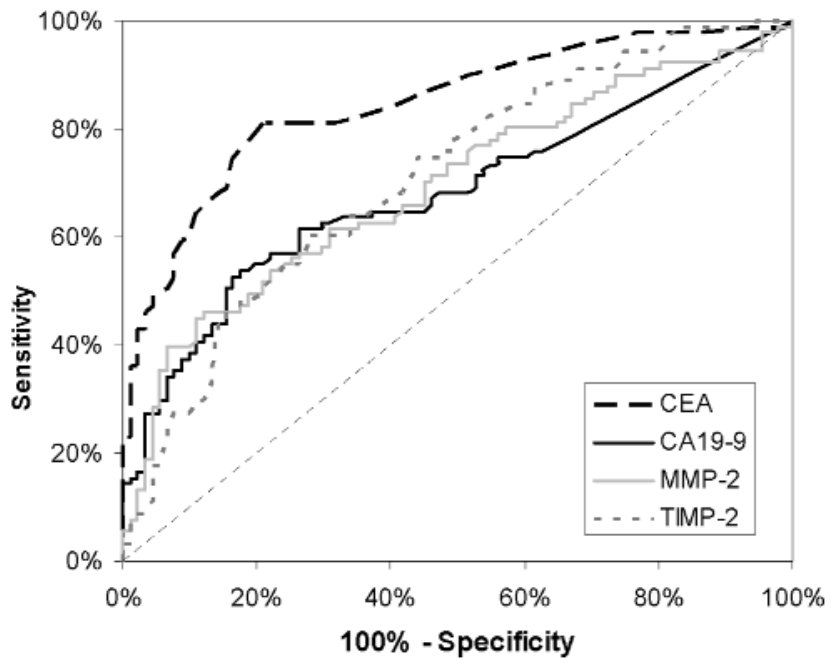

Fig. 2. Areas under ROC curves for CEA (AUC $=0.8473$ ), TIMP-2 $(\mathrm{AUC}=0.7097), \quad \mathrm{MMP}-2 \quad(\mathrm{AUC}=0.6941) \quad$ and $\mathrm{CA} 19-9$ (AUC $=0.6824)$ in differentiation between colorectal cancer patients and healthy subjects

relation analyses. Survival curves were calculated using the method of Kaplan and Meier. Univariate analyses of survival were performed using the log-rank test, and multivariate analyses employed Cox's proportional hazards model. For all multivariate analyses, forward stepwise procedures were used. Differences were considered statically significant with $\mathrm{p}$ values below 0.05 .

Moreover, we calculated diagnostic criteria, such as percentage of true positive levels (diagnostic sensitivity) and area under the receiver operating characteristic (ROC) curve (AUC) for the MMP-2, TIMP-2 and tumor markers. Statistical analyses were carried out using the STATISTICA 9.0 PL program (StatSoft Inc., Tulsa, OK). Diagnostic criteria and the ROC curves were calculated using Med-Calc statistical software (MedCalc Software, Mariakerke, Belgium) and Microsoft Office Excel program (Microsoft Corporation, Redmond, WA).

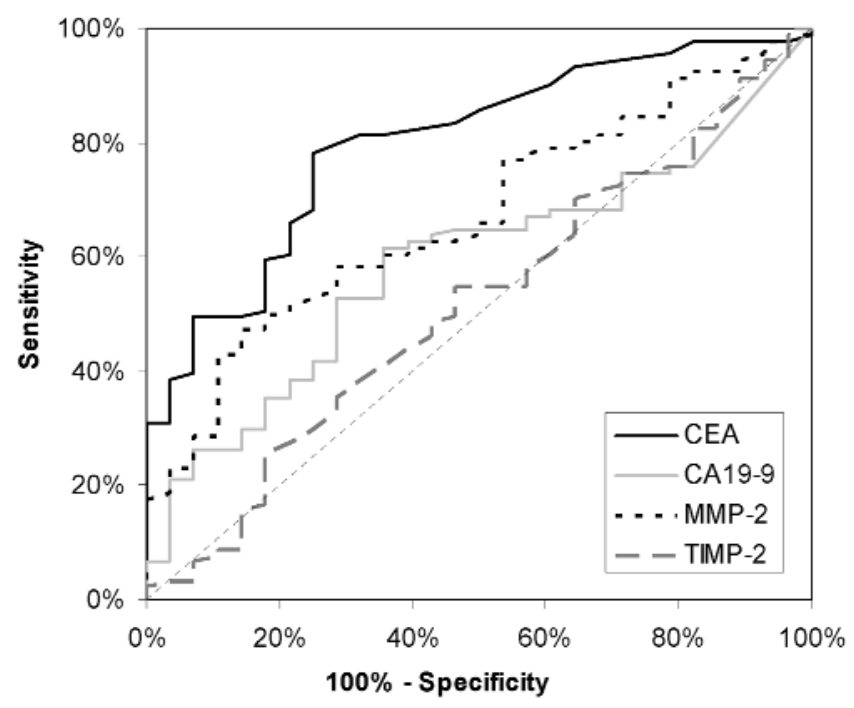

Fig. 3. Areas under ROC curves for CEA ( $\mathrm{AUC}=0.7993$ ), MMP-2 $(\mathrm{AUC}=0.6709), \quad \mathrm{CA} 19-9 \quad(\mathrm{AUC}=0.5893) \quad$ and TIMP-2 $(\mathrm{AUC}=0.5165)$ in differentiation between colorectal cancer and colorectal adenoma patients

\section{Results}

\section{Serum levels of MMP-2, TIMP-2, CEA and CA $19-9$ in colorectal cancer and colorectal adeno- ma patients}

Concentrations of MMP-2, TIMP-2 and tumor markers in the sera of colorectal cancer and adenoma patients as well as in healthy subjects are presented in Table 2. Serum levels of MMP-2 and TIMP-2 were lower, but CEA and CA 19-9 higher in CRC patients than in CA patients and healthy controls. Moreover, levels of MMP-2 and TIMP-2 were lower and tumor 
Table 1. Characteristics of colorectal cancer patients

\begin{tabular}{|c|c|c|}
\hline \multicolumn{2}{|c|}{ Variable tested } & \multirow{2}{*}{$\begin{array}{c}\begin{array}{c}\text { Number of } \\
\text { patients }\end{array} \\
91\end{array}$} \\
\hline \multirow{3}{*}{ Group } & Colorectal cancer & \\
\hline & Colorectal adenoma & 28 \\
\hline & IIcalthy controls & 91 \\
\hline \multirow{6}{*}{$\begin{array}{l}\text { Tumor stage (Dukes } \\
\text { classification) }\end{array}$} & $\mathrm{A}$ & 2 \\
\hline & Bl & 5 \\
\hline & $\mathrm{B} 2$ & 30 \\
\hline & $\mathrm{C} 1$ & 25 \\
\hline & $\mathrm{C} 2$ & 4 \\
\hline & $\mathrm{D}$ & 25 \\
\hline \multirow{4}{*}{$\begin{array}{l}\text { Colorectal wall } \\
\text { infiltration }\end{array}$} & $\mathrm{T} 1$ & 2 \\
\hline & $\mathrm{T} 2$ & 5 \\
\hline & $\mathrm{T} 3$ & 68 \\
\hline & $\mathrm{T} 4$ & 16 \\
\hline \multirow{4}{*}{ Nodal involvement } & No & 40 \\
\hline & N1 & 29 \\
\hline & $\mathrm{N} 2$ & 9 \\
\hline & N3 & 13 \\
\hline \multirow{2}{*}{ Distant metastases } & Mo & 66 \\
\hline & $\mathrm{Ml}$ & 25 \\
\hline \multirow{2}{*}{ Localization of tumor } & Colon & 66 \\
\hline & Reclum & 25 \\
\hline \multirow{2}{*}{ Resectability } & Resectable & 85 \\
\hline & Nonresectable & 6 \\
\hline \multirow{3}{*}{ Patients's survival } & Alive & 49 \\
\hline & Dead & 9 \\
\hline & Data not available & 33 \\
\hline
\end{tabular}

markers higher in adenoma group in comparison with control group. The differences between analyzed groups were significant for all proteins tested in Kruskal-Wallis test. Additionally, the differences between groups were confirmed in Dwass-SteelCritchlow-Fligner post hoc tests and statistical significance was found for comparison of all proteins tested in CRC patients and healthy controls, but for MMP-2 and CEA - in comparison of CRC group with CA patients. The concentrations of MMP-2 and TIMP-2 in patients with colonic cancer were lower, but levels of tumor markers higher than those in rectal cancer subgroup, although the differences were not significant.

Concentrations of MMP-2 and TIMP-2 correlated with tumor stage and were the lowest in the sera of CRC patients with stage $\mathrm{D}$, but serum levels of tumor markers increased and the highest values were observed in patients with most advanced cancer. The differences between cancer stages were significant only for CA 19-9 in Kruskal-Wallis test $(\mathrm{p}=0.027)$ and were confirmed in post hoc Dwass-Steel-CritchlowFligner test for comparison between patients with Duke's stage C and D of CRC patients.

\section{Serum levels of MMP-2, TIMP-2, CEA and CA 19-9 in CRC patients in relation to clinico- pathological features of tumor}

Table 3 presents concentrations of MMP-2, TIMP-2, CEA and CA 19-9 in the sera of CRC patients in relation to clinico-pathological variables of tumor. Serum levels of all proteins tested varied according to bowel wall infiltration (T factor). Concentrations of MMP-2 and TIMP-2 were the lowest but tumor markers the highest in the T4 subgroup, although these differences were not significant. Similar results were observed in serum levels of MMP-2 in relation to nodal involvement - the lowest values were observed in patients with the highest number of metastatic lymph nodes (N3 subgroup), but the differences did not reach statistical significance. In patients with distant metastases (M1 subgroup) concentrations of CEA and CA 19-9 increased significantly in comparison with M0 subgroup ( $\mathrm{p}=0.017$ nad 0.019 , respectively). The concentrations of MMP-2 and TIMP-2 revealed an inverse tendency they decreased in M1 patients, but the differences were not significant in Mann-Whitney test. Moreover, serum levels of MMP-2 and TIMP-2 were lower in CRC patients with nonresectable tumors compared to those with resectable. Similar observations were made in subgroup of CRC patients who died of cancer during observation period, although the differences were not statistically significant. These findings were confirmed in Spearman rank correlation test, where concentrations of MMP-2 significantly correlated with serum levels of TIMP-2 but CEA with CA 19-9.

\section{The diagnostic criteria for MMP-2, TIMP-2, $C E A$ and $C A$ 19-9 in CRC patients}

The percentage of true positive results of proteins tested in CRC patients (diagnostic sensitivity) is presented in Fig. 1. The diagnostic sensitivity of serum TIMP-2 levels $(59 \%)$ was higher than those of MMP-2 (46\%), CEA $(43 \%)$ and CA 19-9 (18\%). The frequency of true positive results was the highest for the combination of CEA with TIMP-2 (79\%). The TIMP-1 area under ROC curve (AUC) for differentiation between CRC and healthy subjects (0.7097) was higher than AUC for MMP-2 (0.6941) and CA 19-9 (0.6824) but lower than for CEA (0.8473) (Fig. 2). Additionally, we analyzed AUC for differentiation between CRC and CA patients. The highest values were obtained for CEA (0.7993) and MMP-2 (0.6709) (Fig. 3). 
Table 2. Serum levels of MMP-2, TIMP-2 and tumor markers in colorectal cancer and adenoma patients

\begin{tabular}{|c|c|c|c|c|c|c|c|c|c|}
\hline \multirow{2}{*}{\multicolumn{2}{|c|}{ Group tested }} & \multicolumn{2}{|c|}{ MMP-2 (ng/mI) } & \multicolumn{2}{|c|}{ TIMP-2 (ng/mI.) } & \multicolumn{2}{|c|}{$\mathrm{CF} \wedge(\mathrm{ng} / \mathrm{mI})$} & \multicolumn{2}{|c|}{$\mathrm{C} \wedge 19-9(\mathrm{TU} / \mathrm{mL})$} \\
\hline & & Median & $\mathrm{p}$ & Median & $\mathrm{p}$ & Median & $\mathrm{p}$ & Median & $\mathrm{p}$ \\
\hline \multicolumn{2}{|l|}{ Colorectal cancer } & $170^{\mathrm{AB}}$ & \multirow{3}{*}{$<0.001^{*}$} & $81^{\mathrm{A}}$ & \multirow{3}{*}{$<0.001^{*}$} & $2.7^{\mathrm{AB}}$ & \multirow{3}{*}{$<0.001^{*}$} & $4.3^{\mathrm{A}}$ & \multirow{3}{*}{$<0.001 \%$} \\
\hline \multicolumn{2}{|l|}{ Colorectal adenoma } & 194 & & $83^{\mathrm{A}}$ & & 0.9 & & 1.4 & \\
\hline \multicolumn{2}{|l|}{ Ilealihy controls } & 205 & & 94 & & 0.8 & & 0.8 & \\
\hline \multirow{3}{*}{ Tumor stage } & $\mathrm{A}+\mathrm{B}$ & 173 & \multirow{3}{*}{0.349} & 82 & \multirow{3}{*}{0.754} & 2.5 & \multirow{3}{*}{0.052} & 3.1 & \multirow{3}{*}{$0.027^{*}$} \\
\hline & $\mathrm{C}$ & 176 & & 80 & & 2.4 & & 2.8 & \\
\hline & D) & 153 & & 77 & & 6.2 & & $13.0^{\circ}$ & \\
\hline \multirow{2}{*}{ Localization of tumor } & Colon & 170 & \multirow{2}{*}{0.572} & 80 & \multirow{2}{*}{0.429} & 2.8 & \multirow{2}{*}{0.821} & 4.3 & \multirow{2}{*}{0.795} \\
\hline & Rectum & 171 & & 81 & & 2.7 & & 2.8 & \\
\hline
\end{tabular}

* statistically significant when $\mathrm{p}<0.05$

A- statistically significant when compared to healthy controls in Dwass-Steel-Critchlow-Fligner post hoc test

B- statistically significant when compared to colorectal adenomas in Dwass-Steel-Critchlow-Fligner post hoc test

C- statistically significant when compared to stage C in Dwass-Steel-Critchlow-Fligner post hoc test

Table 3. Serum levels of MMP-2, TIMP-2 and tumor markers in colorectal cancer patients in relation to clinicopathological features of tumor

\begin{tabular}{|c|c|c|c|c|c|c|c|c|c|}
\hline \multirow{2}{*}{\multicolumn{2}{|c|}{ Group tested }} & \multicolumn{2}{|c|}{$\mathrm{MMP}-2(\mathrm{ng} / \mathrm{mL})$} & \multicolumn{2}{|c|}{ TIMP-2 (ng/mL) } & \multicolumn{2}{|c|}{$\mathrm{CLA}(\mathrm{ng} / \mathrm{mL})$} & \multicolumn{2}{|c|}{ CA19-9 (IU/mL) } \\
\hline & & Median & $\mathrm{p}$ & Median & $\mathrm{p}$ & Median & $\mathrm{p}$ & Median & $\mathrm{p}$ \\
\hline \multirow{3}{*}{ Colorectal wall infiltration $-\mathrm{T}$ factor } & $\mathrm{T} 1+\mathrm{T} 2$ & 175 & \multirow{3}{*}{0.061} & 89 & \multirow{3}{*}{0.147} & 4.2 & \multirow{3}{*}{0.301} & 2.6 & \multirow{3}{*}{0.170} \\
\hline & $\mathrm{T} 3$ & 174 & & 80 & & 2.5 & & 3.4 & \\
\hline & $\mathrm{T} 4$ & 137 & & 77 & & 7.3 & & 9.9 & \\
\hline \multirow{4}{*}{ Nodal involvement $-\mathrm{N}$ factor } & No & 174 & \multirow{4}{*}{0.062} & 82 & \multirow{4}{*}{0.590} & 2.6 & \multirow{4}{*}{0.605} & 3.7 & \multirow{4}{*}{0.093} \\
\hline & N1 & 173 & & 85 & & 2.9 & & 1.7 & \\
\hline & N2 & 204 & & 77 & & 4.5 & & 4.6 & \\
\hline & N3 & 140 & & 79 & & 2.5 & & 6.7 & \\
\hline \multirow{2}{*}{ Distant metastases $-\mathrm{M}$ factor } & M0 & 174 & \multirow{2}{*}{0.298} & 81 & \multirow{2}{*}{0.468} & 2.4 & \multirow{2}{*}{$0.017 *$} & 2.9 & \multirow{2}{*}{$0.019 *$} \\
\hline & Ml & 153 & & 77 & & 6.2 & & 13.0 & \\
\hline \multirow{2}{*}{ Resectability of tumor } & Resectable & 171 & \multirow{2}{*}{0.549} & 81 & \multirow{2}{*}{0.190} & 2.7 & \multirow{2}{*}{0.854} & 4.3 & \multirow{2}{*}{0.541} \\
\hline & Nonresectable & 155 & & 71 & & 3.3 & & 4.5 & \\
\hline \multirow{2}{*}{ Patients's survival } & Alive & 194 & \multirow{2}{*}{0.906} & 77 & \multirow{2}{*}{0.788} & 2.7 & \multirow{2}{*}{0.606} & 4.3 & \multirow{2}{*}{0.803} \\
\hline & Dead & 171 & & 77 & & 3.1 & & 3.7 & \\
\hline
\end{tabular}

* statistically significant when $\mathrm{p}<0.05$

\section{Correlations between MMP-2, TIMP-2 CEA and $C A$ 19-9 serum levels and prognosis of patients' survival}

Univariate analysis showed that tumor stage $(p=0.012)$, presence of nodal $(p=0.015)$ and distant metastases $(\mathrm{p}=0.003)$, tumor resectability $(\mathrm{p}=0.001)$ and serum levels of CEA $(\mathrm{p}=0.007)$ and CA 19-9 $(p=0.007)$ were significant factors affecting overall survival. Age and gender of colorectal cancer patients as well as tumor size ( $\mathrm{T}$ factor) and concentrations of MMP-2 and TIMP-2 were not significant prognostic factors. Multivariate regression analysis with Cox's proportional hazard model revealed that only tumor resectability was an independent prognostic factor for the survival of CRC patients $(\mathrm{p}=0.019)$.

\section{Discussion}

Matrix metalloproteinases, the enzymes involved in mechanisms of tumor progression and formation of distant metastases, may be synthesized by neoplastic and stromal cells [4]. Natural tissue inhibitors of matrix metalloproteinases - TIMPs are induced in stromal 
cells to regulate the reactions of these proteinases [18]. It was suggested that the enhanced cell surface expression of MMPs and TIMPs on inflammatory cells in cancer tissue might be a result of a host response induced by tumors [19]. Increased expression of MMP2 might play a key role in colorectal cancer invasion and/or metastasis $[20,21]$. Moreover, it was shown that in colon and rectal tumor tissues MMP-2 protein levels were higher, while TIMP-2 significantly lower than in corresponding paired normal mucosa [22].

So far, in contrast to tissue expression, little is known about concentrations of MMP-2 and TIMP-2 in the blood of CRC patients. Therefore, this report aims to examine the clinical importance of the measurement of pretreatment serum levels of MMP-2 and TIMP-2 in patients with colorectal adenoma and cancer, especially in the diagnosis of CRC patients as well as in the differentiation between $\mathrm{CA}$ and cancer. The classic tumor markers for CRC - CEA and CA 19-9, which are currently used as biomarkers of tumor burden in clinical practice, were used as reference markers.

In the present study, the preoperative serum levels of CEA and CA 19-9 were significantly higher in colorectal cancer group than in healthy subjects, what is in accordance with our previous results [23]. On the contrary, the serum levels of MMP-2 and TIMP-2 in CRC patients were significantly lower than in healthy subjects. The obtained results are in line with the investigation of Waas et al., who showed that plasma pro-MMP2 levels were lower in colorectal cancer patients than in healthy controls [24]. Moreover, in the study of Oberg et al., the serum level of the MMP-2/TIMP-2 complex was significantly lower in CRC patients as compared to healthy blood donors. However, the serum levels of free MMP-2 and total amount of TIMP-2 were significantly higher in comparison with control group [25]. Additionally, Gehrstein et al. showed the increased content of MMP-2 in tumors in 70-90\% patients with colorectal cancer in comparison with the adjacent normal mucosa, although in their study content of TIMP-2 in tumors did not increase at the level of statistic significance as well as plasma concentrations of both proteins did not correlate with the corresponding values in the tumors and did not surpass the normal levels [26]. It may suggest that in CRC development occur an imbalance between MMP2 and TIMP-2.

In our study, the serum levels of MMP-2 and TIMP-2 correlated with tumor stage and were the lowest in stage $\mathrm{D}$ of cancer, what is in agreement with the results obtained by Larsen et al., who revealed that TIMP-2 concentration in EDTA plasma from CRC patients with Dukes stage A were significantly higher in comparison with more advanced tumor stages [27]. Li et al. demonstrated that with the progression of tumor Duke's stage, the MMP-2/TIMP-2 ratio decreased, suggesting that the balance between MMP-
2 and TIMP-2 plays a crucial role in the invasion of CRC [14]. Additionally, in our paper the serum MMP2 and TIMP-2 levels in CRC patients decreased with bowel wall infiltration ( $\mathrm{T}$ factor), nodal involvement ( $\mathrm{N}$ factor) and presence of distant metastases ( $\mathrm{M}$ factor), as well as in nonresectable tumors, although the differences were not significant. This tendency is opposite to the results of Angenete et al. who revealed that plasma levels of MMP-2 were higher in CRC patients with distant metastases [28]. However, our findings are in accordance with the study of Langenskiöld et al. who found that plasma MMP-2 in CRC patients with T4 tumors was lower than in T3 and T2 subgroups [29]. The decrease of serum MMP-2 and TIMP-2 in more advanced tumor stages might reflect formation of MMP-TIMP complexes in colorectal tumor progression. The obtained results suggest that use of MMP-2 and TIMP-2 might be a predicting tool, especially in combination with different imaging techniques, which might give important preoperative information in staging patients with colorectal cancer.

We also investigated whether the serum concentrations of MMP-2 and TIMP-2 might be useful prognostic factors for CRC patients' survival. The univariate analysis showed that only classic tumor markers, tumor stage, $\mathrm{N}$ and $\mathrm{M}$ factors as well as tumor resectability were significant prognostic factors in CRC patients. Consequently, all these variables included in the multivariate regression analysis, revealed that only tumor resectability may be an independent prognostic factor of CRC patients' survival. Our results are in agreement with those of Waas et al., who showed that the preoperative plasma proMMP-2 levels have no potential value as prognostic markers in CRC. However, they did not investigate the prognostic significance of TIMP-2 [12].

In the present paper, we defined the diagnostic sensitivity and area under ROC curve (AUC) for all the proteins tested to assess a potential clinical significance of MMP-2 and TIMP-2 as tumor markers in the diagnosis of CRC. We revealed that the percentage of true positive results (diagnostic sensitivity) of TIMP-2 (59\%) and MMP-2 (46\%) were higher than frequency of elevated levels of both tumor markers (CEA - 43\%, CA $19-9-18 \%)$. The diagnostic sensitivity of proteins tested was highest for TIMP-2 with CEA $(79 \%)$ and much higher than for combined use of classical tumor markers for CRC - CEA with CA 19-9 (49\%).

The analysis of ROC curves indicates the advantage of TIMP-2 measurement over assessment of classical tumor marker - CA 19-9 in colorectal cancer patients. We found that AUC for TIMP-2 measurement (0.7097) was higher than for MMP-2 - 0.6941 and CA 19-90.6824 but lower than for CEA (0.8473) in differentiation between CRC patients and healthy subjects. The results for CEA and CA 19-9 are similar to those 
obtained in a previous study concerning diagnostic usefulness of classical tumor markers in CRC patients [23]. Additionally, in our study the clinical significance of serum MMP-2 and TIMP 2 in the differentiation between colorectal adenoma and cancer was assessed in the analysis of area under ROC curve for these proteins. The value obtained for MMP-2 (0.6709) was higher than for CA 19-9 and TIMP-2, although lower than for CEA (0.7993) These results suggest a potential clinical usefulness of pretreatment serum MMP-2 for the differentiation between malignant and non-malignant tumors.

In conclusion, the aim of the present study was to investigate the clinical importance of the measurement of serum levels of MMP-2 and TIMP-2 in patients with colorectal adenoma and cancer, especially in the diagnosis of CRC patients as well as in the differentiation between $\mathrm{CA}$ and cancer. We assessed that serum levels of these proteins were lower in CRC patients than in healthy subjects. Moreover, serum MMP-2 was significantly lower in patients with CRC than in colorectal adenoma patients. The percentage of true positive results of serum TIMP-2 was higher than those of other measurands and increased in combined use with CEA. Additionally, the AUC of TIMP-2 was larger than that of MMP-2 in differentiation between CRC and healthy subjects but MMP-2 area was bigger than AUC of tissue inhibitor of matrix metalloproteinases 2 in differentiation between colorectal cancer and adenoma. Our results indicate the better usefulness of TIMP-2 than MMP-2 in the diagnosis of colorectal cancer, especially in combined use with classic tumor marker of CRC, e.g. CEA. Serum MMP-2 seems to be a better tumor marker than TIMP-2 in the differentiation between colorectal cancer and adenoma patients. However, due to discrepancies between the correlation of MMP-2 and TIMP-2 levels with clinico-pathological features of tumor and patients' survival, these findings require further studies.

\section{References:}

[1] Jemal A, Siegel R, Ward E, Hao Y, Xu J, Thun MJ. Cancer statistics, 2009. CA Cancer J Clin. 2009;59:225-49.

[ 2] Fearon ER Molecular genetics of colorectal cancer. Ann NY Acad Sci. 1995;768:101-10.

[3] Fearon ER, Vogelstein B. A genetic model for colorectal tumorigenesis. Cell. 1990; 61:759-67.

[4] Vihinen P, Kahari VM. Matrix metalloproteinases in cancer: prognostic markers and therapeutic targets. Int $J$ Cancer. 2002;99:157-66.

[ 5] Garg P, Sarma D, Jeppsson S, Patel NR, Gewirtz AT, Merlin $\mathrm{D}$, et al. Matrix metalloproteinase-9 functions as a tumor suppressor in colitis-associated cancer. Cancer Res. 2010;70:792-801.

[ 6] Herszényi L, Sipos F, Galamb O, et al. Matrix metalloproteinase-9 expression in the normal mucosa-adenoma-dysplasia-adenocarcinoma sequence of the colon. Pathol Oncol Res. 2008; $14: 31-7$.
[7] Talvensaari-Mattila A, Paakko P, Turpeenniemi-Hujanen T. Matrix metalloproteinase-2 (MMP-2) is associated with survival in breast carcinoma. Br J Cancer. 2003;89:1270-75.

[ 8] Kubben FJ, Sier CF, Meijer MJ, et al. Clinical impact of MMP and TIMP gene polymorphisms in gastric cancer. $\mathrm{Br} J$ Cancer. 2006;95:744-51.

[9] Giannopoulos G, Pavlakis K, Parasi A, et al. The expression of matrix metalloproteinases- 2 and -9 and their tissue inhibitor 2 in pancreatic ductal and ampullary carcinoma and their relation to angiogenesis and clinicopathological parameters. Anticancer Res. 2008;28:1875-81.

[10] Murnane MJ, Cai J, Shuja S, McAneny D, Klepeis V, Willett JB. Active MMP-2 effectively identifies the presence of colorectal cancer. Int J Cancer. 2009;125:2893-902.

[11] Oshima T, Kunisaki C, Yoshihara K, et al. Clinicopathological significance of the gene expression of matrix metalloproteinases and reversion-inducing cysteine-rich protein with Kazal motifs in patients with colorectal cancer: MMP-2 gene expression is a useful predictor of liver metastasis from colorectal cancer. Oncol Rep. 2008;19:1285-91.

[12] Waas ET, Wobbes T, Ruers T, Lomme RM, Hendriks T. Circulating gelatinases and tissue inhibitor of metalloproteinase1 in colorectal cancer metastatic liver disease. Eur J Surg Oncol. 2006;32:756-63.

[13] Wu W, He JT, Ruan JD, Wang RB, Zhang YD. Expression of MMP-2, MMP-9 and collagen type IV and their relationship in colorectal carcinomas. Xi Bao Yu Fen Zi Mian Yi Xue Za Zhi. 2008;24:908-9.

[14] Li BH, Zhao P, Liu SZ, Yu YM, Han M, Wen JK. Matrix metalloproteinase-2 and tissue inhibitor of metallo-proteinase-2 in colorectal carcinoma invasion and metastasis. World J Gastroenterol. 2005;11:3046-50

[15] Shin JE, Jung SA, Kim SE, et al. Expression of MMP-2, HIFlalpha and VEGF in colon adenoma and colon cancer. Korean J Gastroenterol. 2007;50:9-18.

[16] Jass JR, Sobin LH. WHO International Histological Classification of Tumors. Histological Typing of Intestinal Tumors. New York: Springer-Verlag; 1989.

[17] Mroczko B, Szmitkowski M, Okulczyk B. Granulocytecolony stimulating factor (G-CSF) and macrophagecolony stimulating factor (M-CSF) in colorectal cancer patients. Clin Chem Lab Med. 2002;40:351-5.

[18] Sato H, Kida Y, Mai M, et al. Expression of genes encoding type IV collagen-degrading metalloproteinases and tissue inhibitors of metalloproteinases in various human tumor cells. Oncogene. 1992;7:77-83.

[19] Liabakk NB, Talbot I, Smith RA, Wilkinson K, Balkwill F. Matrix metalloprotease 2 (MMP-2) and matrix metalloprotease 9 (MMP-9) type IV collagenases in colorectal cancer. Cancer Res. 1996;56:190-6.

[20] Waas ET, Lomme R-MLM, DeGroot J, Wobbes T, Hendriks $\mathrm{T}$. Tissue levels of active matrix metalloproteinase-2 and -9 in colorectal cancer. Br J Cancer. 2002;86:1876-83.

[21] Waas ET, Wobbes T, Lomme RM, DeGroot J, Ruers T, Hendriks T. Matrix metalloproteinase 2 and 9 activity in patients with colorectal cancer liver metastasis. Br J Surg. 2003;90:1556-64.

[22] Kim TD, Song KS, Li G, et al. Activity and expression of urokinase-type plasminogen activator and matrix metalloproteinases in human colorectal cancer. BMC Cancer. 2006;6:211-21.

[23] Groblewska M, Mroczko B, Wereszczyńska-Siemiątkowska $\mathrm{U}$, et al. Serum interleukin 6 (IL-6) and C-reactive protein (CRP) levels in colorectal adenoma and cancer patients. Clin Chem Lab Med. 2008;46:1423-8.

[24] Waas ET, Hendriks T, Lomme RM, Wobbes T. Plasma levels of matrix metalloproteinase- 2 and tissue inhibitor of metalloproteinase- 1 correlate with disease stage and survival in colorectal cancer patients. Dis Colon Rectum. 2005;48:700-10. 
[25] Oberg A, Höyhtyä M, Tavelin B, Stenling R, Lindmark G. Limited value of preoperative serum analyses of matrix metalloproteinases (MMP-2, MMP-9) and tissue inhibitors of matrix metalloproteinases (TIMP-1, TIMP-2) in colorectal cancer. Anticancer Res. 2000;20:1085-91.

[26] Gershtein ES, Korotkova EA, Prorokov VV, Kushlinsky NE. Matrix metalloproteinases 2, 3, 13 and their type 2 tissue inhibitor in tumors and plasma of patients with colorectal cancer. Bull Exp Biol Med. 2008;145:362-6.

[27] Larsen MB, Stephens RW, Brünner N, et al. Quantification of tissue inhibitor of metalloproteinases 2 in plasma from healthy donors and cancer patients. Scand J Immunol. 2005;61:449-60.
[28] Angenete E, Langenskiöld M, Falk P, Ivarsson ML.Matrix metalloproteinases in rectal mucosa, tumour and plasma: response after preoperative irradiation. Int J Colorectal Dis. 2007;22:667-74.

[29] Langenskiöld M, Holmdahl L, Falk P, Ivarsson ML. Increased plasma MMP-2 protein expression in lymph node-positive patients with colorectal cancer. Int $J$ Colorectal Dis. 2005;20:245-52.

Submitted: 5 July, 2010

Accepted after reviews:5 August, 2010 